\title{
Amisulpride plus valproate vs haloperidol plus valproate in the treatment of acute mania of bipolar I patients: a multicenter, open-label, randomized, comparative trial
}

\author{
Pierre Thomas' \\ Eduard Vieta ${ }^{2}$ \\ for the SOLMANIA study \\ group \\ 'Department of Psychiatry, Fontan \\ Hospital CHRU Lille, University of \\ Lille 2, France; ${ }^{2}$ Bipolar Disorders \\ Program, Hospital Clinic, University \\ of Barcelona, IDIBAPS, CIBERSAM, \\ Barcelona, Spain
}

\begin{abstract}
The primary objective of this study was to compare the effectiveness of combination treatment of valproate and amisulpride with that of valproate and haloperidol in bipolar I disorder. Adult inpatients with a current manic episode fulfilling DSM-IV-TR diagnostic criteria for bipolar type I disorder were included. Patients were randomized to amisulpride (400-800 mg/day) or haloperidol (5-15 mg/day) for 3 months and all received valproate. The primary effectiveness criterion was the percentage of responders (defined by a decrease of $\geq 50 \%$ of the Y-MRS) in patients completing the study. Safety was evaluated by adverse event reporting, determination of extrapyramidal function and clinical examination. Sixty-two patients were randomized to receive valproate-amisulpride, and 61 to receive valproate-haloperidol. At study end, responder rates were $72.6 \%$ in the amisulpride group and $65.5 \%$ in the haloperidol group. Remission rates were $83.9 \%$ and $89.7 \%$, respectively. At study end, neither response rates nor remission rates differed significantly between groups. Treatment-emergent adverse events occurred significantly $(\mathrm{p}=0.009)$ more frequently in the haloperidol group $(86.4 \%)$ than in the amisulpride group $(66.1 \%)$. In conclusion, the valproate-amisulpride combination was as effective as the valproate - haloperidol combination in bipolar I patients, with a better safety profile.
\end{abstract}

Keywords: amisulpride, valproate, haloperidol, clinical trial, mania, bipolar disorder

\section{Introduction}

Bipolar I disorder is an episodic, lifelong illness characterized by alternating phases of mania and depression. The lifetime prevalence of bipolar type I disorder estimated in general population surveys conducted in Europe ranges from $0.3 \%$ to $1.8 \%$ (Pini et al 2005). Bipolar disorder carries with it an increased risk of psychiatric or somatic morbidity (Krishnan 2005), substance abuse (Levin and Hennessy 2004), and suicide (Angst et al 2005), and is highly detrimental to stable social functioning (Elgie and Morselli 2007).

Current practice guidelines recommend first-line treatment of acute mania with either lithium, valproate, or an atypical antipsychotic (APA 2002; Goodwin 2003; Grunze et al 2003). As monotherapy, atypical antipsychotics show similar efficacy to mood stabilizers (lithium or valproate) (Smith et al 2007), whereas combination of the two classes of drug brings superior efficacy (Scherk et al 2007) and is considered useful in cases of severe mania or inadequate response to monotherapy. Combining antipsychotic drugs with lithium or valproate achieves a more rapid onset of response, better control of agitation and insomnia, and a lower risk of rebound depression (Bowden 2005; Scherk et al 2007). Although haloperidol has been used widely for the acute treatment of mania, its use is associated with a higher incidence of extrapyramidal 
effects than the more recent atypicals and may carry a higher risk of switch to depression and poor adherence (Tohen et al 2003; Smulevich et al 2005).

Nonetheless, few randomized clinical trials have compared atypical with classical antipsychotics in the treatment of acute mania. Sachs and colleagues (Sachs et al 2002) compared treatment with a combination of haloperidol and a mood-stabilizer with a combination of risperidone and a mood stabilizer and to a mood stabilizer alone in a 3-week study. Both antipsychotic combinations were superior to mood stabilizer alone in terms of symptomatic control of mania, with no significant difference being observed between them. However, extrapyramidal side-effects were observed significantly more frequently with haloperidol than with the other two study treatments. In addition, a number of randomized studies have compared haloperidol monotherapy with olanzapine monotherapy (Shi et al 2002; Tohen et al 2003), quetiapine (McIntyre et al 2005), risperidone (Segal et al 1998; Smulevich et al 2005), and aripiprazole (Vieta et al 2005a) in acute mania. The majority of these have demonstrated comparable response or remission rates with the atypical antipsychotic to haloperidol, with the exception of the aripiprazole study. However, response rates to haloperidol in this study were particularly low compared with other studies, because the definition of response included the requirement to stay on medication for 12 weeks. A metaanalysis of these studies showed similar reduction of manic symptoms and comparable response rates for haloperidol and for the atypical antipsychotics, but lower dropout rates due to adverse events, lower incidence of extrapyramidal symptoms, and better control of depressive symptoms with the atypical antipsychotics (Scherk et al 2007).

Amisulpride is an atypical antipsychotic which has been suggested to show superior efficacy to both haloperidol and risperidone in improving affective symptoms in patients with schizophrenia (Peuskens et al 2002) and for which there is some evidence for efficacy in affective disorders (Montgomery 2002). A prospective open-label study of amisulpride as add-on therapy to mood stabilizers in the treatment of acute mania reported by Vieta et al showed a 3-fold reduction in YMRS total scores after 6 weeks treatment with adjunctive amisulpride. Sixty-five percent of patients were considered responders (at least 50\% improvement of YMRS scores) and remission was achieved by $50 \%$ of patients (Vieta et al $2005 \mathrm{~b}$ ). Also, a longitudinal study evaluating relapse rates before and after the introduction of amisulpride in patients treated for bipolar I disorder showed a 3-fold reduction of relapse rates after the introduction of amisulpride (Carta et al 2006).
Amisulpride also shows a low incidence of extrapyramidal side-effects (Leucht et al 2002) and less propensity to produce weight gain compared with some other atypical antipsychotic (Leucht et al 2002). Therefore, combination treatment with amisulpride and a mood-stabilizer may be of interest in the treatment of bipolar disorder. In order to test this hypothesis, we have performed a randomized, open-label clinical trial comparing amisulpride and valproate combination therapy with haloperidol and valproate combination therapy in the treatment of acute mania.

The primary objective of this study was to compare the effectiveness of valproate and amisulpride combination treatment with that of valproate and haloperidol combination treatment for 3 months in patients with type I bipolar disorder presenting with a manic episode. Secondary objectives were to evaluate the clinical and biological safety of the two combination treatments, to evaluate efficacy after 3 weeks, and to assess patient satisfaction at 3 months.

\section{Subjects and methods Study design}

This was a multinational, randomized, open-label, parallelgroup, comparative phase II study. The study duration was 3 months. The study was conducted in 27 centers in 5 European countries (Czech Republic, France, Poland, Slovakia, and Spain) between May 2004 and June 2005.

\section{Entry criteria}

The study included subjects aged 18-65 years with a current diagnosis of bipolar I disorder according to DSM-IV-TR criteria (APA 2000). Subjects were required to be currently in a manic episode fulfilling DSM-IV-TR criteria and to have experienced at least 1 manic episode in the past. The total score on the Young Mania Rating Scale (YMRS; Young et al 1978) was to be at least 20, with a score of at least 3 for at least 2 of the following YMRS items: elevated mood, increased motor activity, energy, sleep, or content (grandiosity). The score on the severity scale of the Clinical Global Impression for Bipolar Disorder questionnaire (CGI-BP; Spearing et al 1997) was to be at least 5.

Women of childbearing age were expected to be using an effective method of contraception and all subjects had to be able to comply with the study protocol. Pregnant and breast-feeding women were excluded.

Subjects with antecedents of intolerance to benzamide drugs, haloperidol or valproate, contraindications to any of these drugs, with comorbidities that might affect implementation or interpretation of the study, with clinically relevant 
abnormalities in biochemical (notably hypokalemia, transaminases higher than twice the upper limit of normal, or bilurubin or alkaline phosphatase outside the normal range), hematological or electrocardiographic (in particular, heart rate $<55$ bpm, congenital QT prolongation, or QTc Bazett interval $>450 \mathrm{~ms}$ in males and $>470 \mathrm{~ms}$ in females) parameters, those having participated in a clinical trial within the previous 3 months, and subjects with current or recent substance abuse or dependence were not included in the study.

Treatment with fluoxetine in the previous 4 weeks, with injectable long-acting antipsychotics if the interval between two injection periods had not elapsed at inclusion, with a mood stabilizer other than valproate at an effective dose for less than 7 days preceding the screening visit, and for whom a treatment switch was not justified, was not allowed. Other prohibited drugs included Class Ia and Class III antiarrythmic drugs, beperidil, cisapride, sultopride, thioridrazine, erythromycin (iv), vincamine (iv), halofantril, pentamidine, and sparfloxacin.

\section{Study procedures}

Subjects fulfilling the entry criteria were randomized to receive either amisulpride and valproate or haloperidol and valproate. During the screening visit, the investigator contacted the contract research organization in charge of the operational management of the study by telefax in order to obtain a patient treatment number. Each number corresponded to one or other of the study treatments. Study medication was provided in numbered containers divided into separate packs containing sufficient drug to last the patient between 2 consecutive study visits, the total quantity covering the entire study period. Randomization was stratified by country and numbers allocated on a patient by patient basis rather than in blocks. The randomization code was generated and maintained centrally by the contract research organization in charge of the operational management of the study. Patients were randomized into the study and allocated study treatment in a sequential order according to this list, in each country. The allocation sequence was not revealed before treatments had been assigned.

The treatment phase was preceded by a 3-day wash-out period which could be reduced to 1 day in case of aggravation of mania. The treatment phase lasted for 12 weeks. Subjects were hospitalized during the washout period and for at least the 2 first weeks of the active treatment period; hospital discharge was decided when considered appropriate by the investigator. Study visits were scheduled on Day -3, Day 0, Day 4, Day 7, Week 2, Week 3, Week 8, and
Week 12. Evaluations made at Week 3 and Week 12 were used for the efficacy evaluation. A latitude of 3 days and 7 days respectively was allowed for these study visits either side of the planned date. Additional visits could be made if considered appropriate by the investigator.

\section{Treatment}

Amisulpride was provided as scored $400 \mathrm{mg}$ tablets (Solian ${ }^{\circledR}$, Sanofi-Aventis, Paris, France) and haloperidol as scored $5 \mathrm{mg}$ tablets. Valproate was provided as the pharmaceutical form approved for the treatment of bipolar disorder in participating countries (sustained-release sodium valproate + valproic acid tablets $500 \mathrm{mg}$ for the Czech Republic, Poland, Slovakia, and Spain, or enteric-coated valproate semisodium tablets $500 \mathrm{mg}$ or $250 \mathrm{mg}$ in France). No blinding of study medication was attempted in order to simplify the procedures and to enrol a more representative sample. Treatment was initiated at a dose of $600 \mathrm{mg}$ /day for amisulpride and $10 \mathrm{mg}$ /day for haloperidol. In both cases, the starting dose of valproate used was that specified in local prescribing recommendations $(10-15 \mathrm{mg} / \mathrm{kg}$ in Czech Republic, $20 \mathrm{mg} / \mathrm{kg} /$ day in France, $5-15 \mathrm{mg} / \mathrm{kg}$ in Poland, $10-15 \mathrm{mg} / \mathrm{kg}$ in Slovakia, $20-30 \mathrm{mg} / \mathrm{kg} /$ day in Spain). From Day 4 onwards, the dose could be adjusted at the investigator's discretion at any study visit, as a function of the clinical state of the subject. For amisulpride, dose adjustment was made in steps of $200 \mathrm{mg}$ within a range of 400-800 mg/day. For haloperidol, the adjustment was made in steps of $5 \mathrm{mg}$ within a range of 5-15 mg/day. Dose adjustments were made on the day after the study visit and could be made as many times as deemed necessary. The dose of valproate could be adjusted accordingly if plasma levels were outside the $40-100 \mu \mathrm{g} / \mathrm{mL}$ range at the Week 3 study visit, or in case of emergence of side-effects. Otherwise, the dose of valproate was not changed during the course of the study. Treatment was pursued for 12 weeks.

At each study visit, the patient was expected to return the previous medication pack and receive a new one covering the period until the next study visit. A pill count of returned medication was made, and the number of returned pills recorded on a drug tracking form. On this basis, the investigator evaluated reported the overall compliance of the patients at each study visit on the case report form (yes or no).

Concomitant use of other psychotropic drugs was prohibited, except for nonbenzodiazepine hypnotics in case of insomnia and lorazepam if needed to control agitation, irritability, restlessness, insomnia, and hostile behaviors. The maximum daily dose of lorazepam was $6 \mathrm{mg}$ in the first week and $2 \mathrm{mg}$ in the second and third weeks. Thereafter 
benzodiazepine use was prohibited. Antiparkinsonian drugs (eg, biperiden or procyclidine or tropatepine) could be introduced to control incapacitating extrapyramidal symptoms documented by the Simpson Angus Scale (Simpson and Angus 1970). The use of other drugs entering in the exclusion criteria for the trial (eg, anti-arrhythmic drugs) was also prohibited.

\section{Evaluation of effectiveness}

The primary efficacy criterion was the proportion of subjects fulfilling a combined criterion of response, defined as a reduction of at least $50 \%$ of the YMRS score, and completion of the 12-week treatment period. This outcome was chosen because the trial was aimed at assessing effectiveness, rather than pure efficacy, and because it has been successfully used before (Vieta et al 2005a). Secondary efficacy criteria included the proportion of responders at Weeks 3 and 12, the proportion of subjects in remission, defined as a YMRS score $\leq 12$, at study end, changes from baseline in YMRS score and Brief Psychiatric Rating Scale (BPRS; Overall and Gorham 1962) score at Weeks 3 and 12, changes from baseline in the Montgomery and Åsberg Depression Rating Scale (MADRS; (Montgomery and Åsberg 1979)) and the Global Assessment Scale (GAS; Endicott et al 1976) at study end and the change item and patient satisfaction item of the CGI-BP at study end. The maintenance of response was evaluated by Kaplan-Meier survival analysis. A sustained response was defined as reaching a YMRS score $\leq 12$ and a MADRS score of $\leq 15$ for at least 2 consecutive visits. Subjects switching to depression or relapsing into mania were identified. A switch to depression was defined as an increase in the CGI-BP depression score of at least 2 points and a MADRS score $\geq 15$. A relapse into mania was defined as an increase of at least $25 \%$ in the YMRS total score following a sustained response. A posthoc analysis of efficacy was performed on patients with prominent psychosis defined by a score of at least 3 for the "Content" item of the YMRS scale at D0, or a score of at least 4 for at least 2 of the 5 following items of the BPRS scale at D0 (conceptual disorganization, grandiosity, suspiciousness, hallucinatory behavior, and unusual thought content). A second posthoc analysis was also performed on patients with depressive symptoms at inclusion defined by a score at baseline of at least 10 on the MADRS scale.

\section{Evaluation of safety}

Safety was assessed by recording spontaneously reported adverse events throughout the study. Adverse events were classified according to severity and to presumed relationship with treatment. Extrapyramidal function was assessed with the Simpson Angus scale (SAS), the Barnes akathisia scale (Barnes 1989) at each study visit, and the Abnormal Involuntary Movement Scale (AIMS; NIMH 1976). Vital signs and body weight were measured at each visit. Standard laboratory tests were performed before the first treatment administration, at Weeks 3, 8, and 12. Treatment compliance to treatment was assessed by the investigator by counting unused tablets.

\section{Statistical analysis}

The statistical analysis was designed to test the hypothesis that the effectiveness of combination treatment with amisulpride and valproate would be superior to that of combination treatment with haloperidol and valproate after 3 months. To this end, a priori power calculations were performed to determine the target population size. A difference between treatment groups in the proportion of subjects meeting the primary endpoint of $22 \%$ was considered to be the minimum clinically relevant difference based on a previous trial with aripiprazole (Vieta et al 2005a; Vieta and Carne 2005). It was estimated that, in order to have a power of $80 \%$ to demonstrate such a difference with a one-tailed $\alpha$-risk of $0.05,60$ subjects should be included in each treatment arm.

Three study populations were considered. The safety population was defined as all subjects exposed to at least 1 dose of study medication. The intention-to-treat (ITT) population was defined as all randomized subjects who took at least one dose of study medication and provided at least one postbaseline efficacy measure. The per protocol (PP) population was defined as those members of the ITT population who did not present a major protocol violation. Since this was a superiority study, the ITT population was used for determination of effectiveness, as this is the most conservative assumption. Supportive analyses were performed on the PP population. Missing data were handled by the principle of last observation carried forward (LOCF).

Categorical variables were compared with the $\chi^{2}$ test or Fisher's exact test and quantitative variables with the Wilcoxon test or by analysis of variance (ANOVA). Two-tailed comparisons were performed throughout, with a probability (p) value of 0.05 taken as significant. Statistical analyses were carried out using the SAS software package (Version 8.2; SAS Institute, Cary, NC, USA).

\section{Ethics}

The study was conducted according to the Declaration of Helsinki (Hong Kong Amendment), Good Clinical Practices 
(European Guidelines), and pertinent national legal and regulatory requirements. Written informed consent was obtained from each subject. Subjects were free to withdraw from the study at any time for any reason, without effect on their medical care. The protocol was submitted to and approved by the appropriate local ethics committee in each country.

\section{Results}

\section{Study population}

One hundred and twenty-six subjects were screened for inclusion and the 123 who fulfilled the entry criteria were randomized to 1 of the 2 treatment arms, 62 to amisulpride, and 61 to haloperidol. The median number of patients included by center was 5 (range: 1-25). Two subjects randomized to the haloperidol group never received the study medication and were excluded from the safety population. An additional subject in this group provided no postbaseline efficacy data and was thus excluded from the ITT population. The ITT population thus consisted of 62 subjects in the amisulpride arm and 58 subjects in the haloperidol arm. Major protocol deviations were detected in 15 subjects in the amisulpride group and in 3 subjects in the haloperidol group, who were excluded from the PP population. The PP population corresponded to 102 subjects overall, $75.8 \%$ of the group originally randomized to amisulpride and $90.2 \%$ of the group originally randomized to haloperidol. The major protocol violations detected mostly concerned deviations from the permitted time windows for study visits at Weeks 3 and 12 (10 violations in the amisulpride group and 2 in the haloperidol group) or use of prohibited psychotropic medication (6 in the amisulpride group; Table 1). Fifteen subjects in the amisulpride group and 23 in the haloperidol group failed to complete the 12-week treatment period as planned, principally due to the occurrence of an adverse event ( 7 subjects in the amisulpride group and 15 in the haloperidol group; Table 1). Subject flow through the study is presented in Figure 1.

The demographic and clinical characteristics of the ITT population are presented in Table 2. None of these variables differed significantly between the two treatment groups, with the exception of mood state at entry. The mean age of the population was $43.6 \pm 12.7$ years and the majority $(59.2 \%)$ were female. Overall, subjects had a long history of bipolar disorder (median disease duration: 11 years [range: 1-38 years]; median number of previous episodes: 4 [range: 1-40]). At inclusion, the mood state was euphoric in all but 3 subjects in the amisulpride group and 14 in the haloperidol group. This was the only significant intergroup difference observed in the ITT population $(\mathrm{p}=0.003$;
Table I Major protocol violations and premature study discontinuations in the ITT population. Note that individual subjects may present more than one protocol violation or reason for discontinuation and the categories are thus not mutually exclusive

\begin{tabular}{|c|c|c|}
\hline & $\begin{array}{l}\text { Amisulpride }+ \\
\text { valproate } \\
(\mathbf{N}=\mathbf{6 2})\end{array}$ & $\begin{array}{l}\text { Haloperidol + } \\
\text { valproate } \\
(\mathbf{N}=\mathbf{5 8})\end{array}$ \\
\hline Major protocol violations & 15 & 3 \\
\hline $\begin{array}{l}\text { Week } 3 \text { visit time window } \\
\text { not respected }\end{array}$ & 3 & 0 \\
\hline $\begin{array}{l}\text { Week } 12 \text { visit time window } \\
\text { not respected }\end{array}$ & 7 & 2 \\
\hline No BPRS data at baseline & - & 1 \\
\hline No GAS data at baseline & - & 1 \\
\hline $\begin{array}{l}\text { Use of prohibited psychotropic } \\
\text { medication (psychotropic } \\
\text { agents other than hypnotics and } \\
\text { benzodiazepines } \geq 72 \text { hours) }\end{array}$ & 6 & 0 \\
\hline $\begin{array}{l}\text { Premature treatment } \\
\text { discontinuations }\end{array}$ & 15 & 23 \\
\hline $\begin{array}{l}\text { Occurrence of an adverse } \\
\text { event }\end{array}$ & 7 & 15 \\
\hline Perceived lack of efficacy & 4 & 5 \\
\hline Consent withdrawn & 3 & 6 \\
\hline Poor compliance & I & I \\
\hline Other & I & 2 \\
\hline
\end{tabular}

Abbreviations: BPRS, Brief Psychiatric Rating Scale GAS, Global Assessment Scale.

Fisher's exact test). The mean mania score on the YMRS was 29.9. Psychiatric comorbidity was present in 9 subjects in each group, namely substance abuse (principally alcohol or cannabis) in 5 subjects in the amisulpride group and 7 in the haloperidol group, anxiety disorders in 5 and 2 subjects respectively (one amisulpride-treated subject presented both disorders).

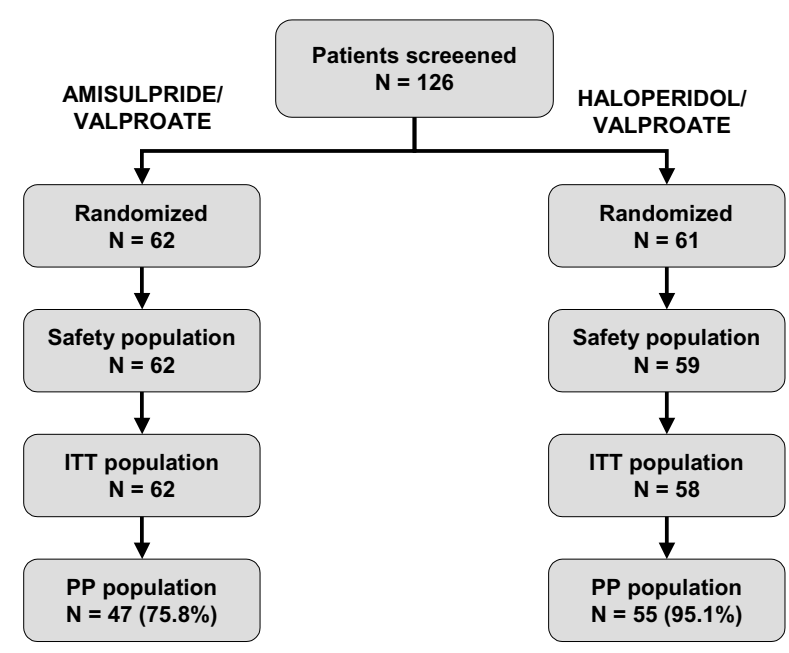

Figure I Subject flow diagram. Abbreviations: ITT, intention to treat; PP, per protocol. 
Table 2 Baseline demographic and clinical characteristics of subjects in the intention to treat population. Data for categorical variables are presented as numbers of subjects (\%) and quantitative variables as mean \pm standard deviation

\begin{tabular}{|c|c|c|}
\hline & $\begin{array}{l}\text { Amisulpride }+ \\
\text { valproate } \\
(\mathbf{N}=62)\end{array}$ & $\begin{array}{l}\text { Haloperidol + } \\
\text { valproate } \\
(\mathbf{N}=\mathbf{5 8})\end{array}$ \\
\hline Gender (female) & $36(58.1 \%)$ & $35(60.3 \%)$ \\
\hline Age (years) & $41.8 \pm 12.9$ & $45.4 \pm 12.3$ \\
\hline Body mass index $\left(\mathrm{kg} / \mathrm{m}^{2}\right)$ & $26.3 \pm 5.4$ & $26.0 \pm 4.9$ \\
\hline $\begin{array}{l}\text { Duration of bipolar disorder } \\
\text { (years) }\end{array}$ & $12.2 \pm 8.9$ & $13.0 \pm 8.8$ \\
\hline $\mathrm{Nr}$ of previous episodes & $7.2 \pm 8.6$ & $6.2 \pm 5.5$ \\
\hline $\mathrm{Nr}$ of previous manic episodes & $3.8 \pm 5.0$ & $4.5 \pm 4.7$ \\
\hline Time since last episode (years) & $1.8 \pm 1.8$ & $1.8 \pm 2.1$ \\
\hline \multicolumn{3}{|l|}{ Type of the last episode } \\
\hline Manic episode & $4 \mathrm{l}(66.1 \%)$ & $43(74.1 \%)$ \\
\hline Hypomanic episode & $\mathrm{I}(\mathrm{I} .6 \%)$ & $\mathrm{I}(\mathrm{I} .7 \%)$ \\
\hline Mixed episode & $2(3.2 \%)$ & $\mathrm{I}(\mathrm{I} .7 \%)$ \\
\hline Major depressed episode & $18(29.0 \%)$ & $13(22.4 \%)$ \\
\hline Psychiatric comorbidity & $9(14.5 \%)$ & $9(15.5 \%)$ \\
\hline Past psychotropic drug use $\mathrm{e}^{\mathrm{a}}$ & $54(87.1 \%)$ & $5 \mathrm{I}(87.9 \%)$ \\
\hline Previous hospitalization & 51 (82.3\%) & $54(93.1 \%)$ \\
\hline \multicolumn{3}{|l|}{ Mood state at inclusion } \\
\hline \multicolumn{3}{|l|}{ into study } \\
\hline Dysphoric mania & $3(4.8 \%)$ & 14 (24.1\%) \\
\hline Euphoric mania & 59 (95.2\%) & $44(75.9 \%)$ \\
\hline YMRS score & $29.2 \pm 5.2$ & $30.5 \pm 5.3$ \\
\hline BPRS score & $35.8 \pm 9.4$ & $37.7 \pm 9.9$ \\
\hline GAS score & $43.5 \pm 10.0$ & $43.8 \pm 11.2$ \\
\hline MADRS score & $8.7 \pm 3.1$ & $9.4 \pm 3.7$ \\
\hline \multicolumn{3}{|l|}{ CGI-BP Severity score } \\
\hline Mania & $5.3 \pm 0.5$ & $5.4 \pm 0.5$ \\
\hline Depression & $1.2 \pm 0.8$ & $1.2 \pm 0.6$ \\
\hline General bipolar & $4.8 \pm 1.0$ & $5.2 \pm 0.9$ \\
\hline
\end{tabular}

aln previous 6 months.

Abbreviations: BPRS, Brief Psychiatric Rating Scale; CGI-BP, Clinical Global Impression Bipolar Sale; GAS, Global Assessment Scale; MADRS, Montgomery and Åsberg Depression Rating Scale; YMRS, Young Mania Rating Scale.

The subgroup of patients with prominent psychosis consisted of 87 subjects ( $72.5 \%$ of the ITT population). At inclusion, the mean mania score on the YMRS in this subgroup was 30.9 , with mean scores for amisulpride $(n=46)$ and haloperidol $(n=41)$ of 30.8 and 31.1 respectively. The subgroup of patients with depressive symptoms at inclusion defined by MADRS score of at least 10 consisted of 50 patients with 23 patients in the amisulpride group and 27 patients in the haloperidol group.

The majority of patients (all but 8 in the amisulpride group and 7 in the haloperidol group) had received psychotropic medication in the previous 6 months. Eleven patients (9.2\%) had received lithium, 15 (12.5\%) carbamazepine or oxcarbazepine, and $52(43.3 \%)$ valproate for control of mood disorders. The response to these treatments was not recorded. In addition, 58 patients (48.3\%) had received atypical antipsychotics, $53(44.2 \%)$ classical antipsychotics, 49 (40.8\%) benzodiazepines, $26(21.7 \%)$ antidepressants, and 17 (14.2\%) nonbenzodiazepine hypnotics. No difference in previous psychotropic drug exposure was observed between treatment groups.

\section{Exposure to medication}

The dose of study medication evolved over the study, generally downwards. At the end of the study around half of subjects treated with amisulpride were receiving a dose of $400 \mathrm{mg}$ /day and over three-quarters of those in the haloperidol group were receiving a dose of $5 \mathrm{mg}$ /day (Figure 2). However, the dose of amisulpride was increased to $800 \mathrm{mg}$ in around one-fifth of the subjects. Overall compliance to study medication was considered to be acceptable in all patients and at each study visit with the exception of 1 subject in the amisulpride group on Day 4, 3 subjects in the amisulpride group at Month 3, and 4 subjects in the haloperidol group at Month 3.

At Week 3, plasma concentrations of valproate were significantly lower in the amisulpride group $(64.5 \mu \mathrm{g} / \mathrm{mL}$ [95\% confidence intervals (CI): 57.2-71.9]) than in the haloperidol group $(74.9 \mu \mathrm{g} / \mathrm{mL}$ [95\% CI: 67.5-82.4]). However, this difference was no longer apparent at Week 12 (amisulpride: $64.3 \mu \mathrm{g} / \mathrm{mL}$ [48.8-79.7]; haloperidol: $69.2 \mu \mathrm{g} / \mathrm{mL}$ [61.9-76.6]).

For concomitant psychotropic medications taken by the patients during the course of the trial, no statistical differences were shown between the two treatment groups with regard to either benzodiazepine $(\mathrm{p}=0.944)$ or hypnotic $(\mathrm{p}=0.659)$ use. Authorized benzodiazepines were used by $58.3 \%$ of subjects at inclusion, with no difference between groups (35 subjects in each group). Benzodiazepine use declined thereafter over the course of the study. Antiparkinsonian drugs were prescribed to 12 subjects (19.4\%) in the amisulpride group and
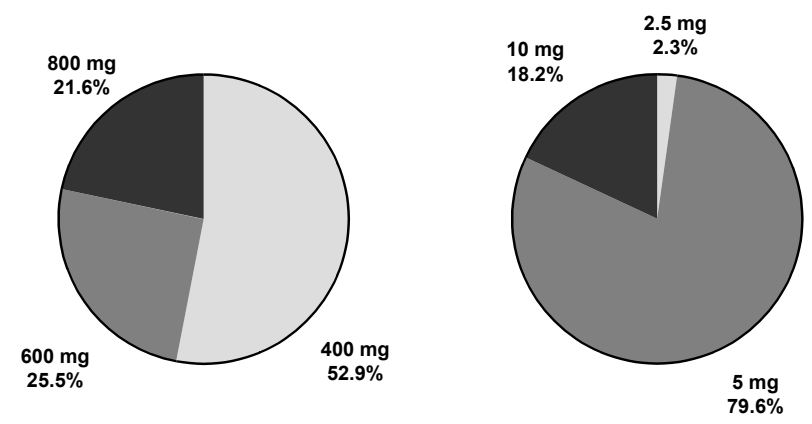

Figure 2 Treatment dose at study end. Left: amisulpride; right: haloperidol. 
to $32(54.2 \%)$ in the haloperidol group (see Safety section below).

\section{Effectiveness}

The primary outcome measure was the proportion of subjects who completed the 3-month study period and responded to treatment ( $\geq 50 \%$ reduction in YMRS score compared with baseline) in the ITT population. The number of subjects fulfilling this criterion was $45 / 62(72.6 \%)$ in the amisulpride group and $38 / 58(65.5 \%)$ in the haloperidol group. These proportions were not significantly different $\left(\mathrm{p}=0.402 ; \chi^{2}\right.$ test). The corresponding figures for the PP population were 35/47 (74.5\%) in the amisulpride group and $36 / 55(65.5 \%)$ in the haloperidol group ( $\mathrm{p}=0.324 ; \chi^{2}$ test). In the subgroup of patients with prominent psychosis, the number of subjects fulfilling the primary endpoint was 35/46 (76.1\%) in the amisulpride group and $27 / 41(65.9 \%)$ in the haloperidol group, with no significant difference between groups ( $\mathrm{p}=0.292 ; \chi^{2}$ test).

No significant differences between treatment groups were observed for remission rates, response rates, or mean change in YMRS scores. At the end of the 12-week treatment period, 52 subjects in the amisulpride group (83.9\%) and 52 subjects in the haloperidol group $(89.7 \%)$ were considered to be in remission (YMRS score $\leq 12$ ); this difference was not statistically significant. Response rates at study end were $87.1 \%$ (54/62 subjects) in the amisulpride group and 93.1\% (54/58 subjects) in the haloperidol group. Mean YMRS scores declined rapidly in both groups with no obvious difference in the rate of response (Figure 3). During the course of the study $74.2 \%$ of the amisulpride group (46/62 subjects) and $84.5 \%$ of the haloperidol group (49/58 subjects) achieved a sustained response. One subject in the amisulpride group and 6 subjects in the haloperidol group relapsed into mania after having achieved a sustained response, all of whom belonged to the subgroup of patients with prominent psychosis. There was a trend toward a lower risk of relapse for patient treated by amisulpride during the 3-month treatment period ( $\mathrm{p}=0.055)$. A switch to depression was observed in 6 subjects in the amisulpride group and in 10 subjects in the haloperidol group $(p=0.223)$. The percentage of subjects switching to depression was equivalent for subgroups of subject with a MADRS score at inclusion $\geq 10$ or $\leq 10$.

The mean change in YMRS score was comparable in the two treatment groups at both Week 3 and Week 12 (Table 3). Similarly, scores on all the other psychopathology rating scales improved to a similar extent in both treatment groups (Table 3). Changes on the depression item of the CGI-BP and the MADRS were, however, minimal. No statistically significant differences between treatment groups were identified for any of these secondary end-points. However, a trend was observed for a greater decrease of MADRS scores between baseline and study end in the amisulpride group (mean percentage change in MADRS score: $-27.5 \%$ vs $-0.3 \%$ ).

Around three-quarters of subjects considered themselves much improved or very much improved at study end (Figure 4). No significant difference in CGI-BP Change item category distribution was observed between the two treatment groups $\left(\chi^{2}\right.$ test).

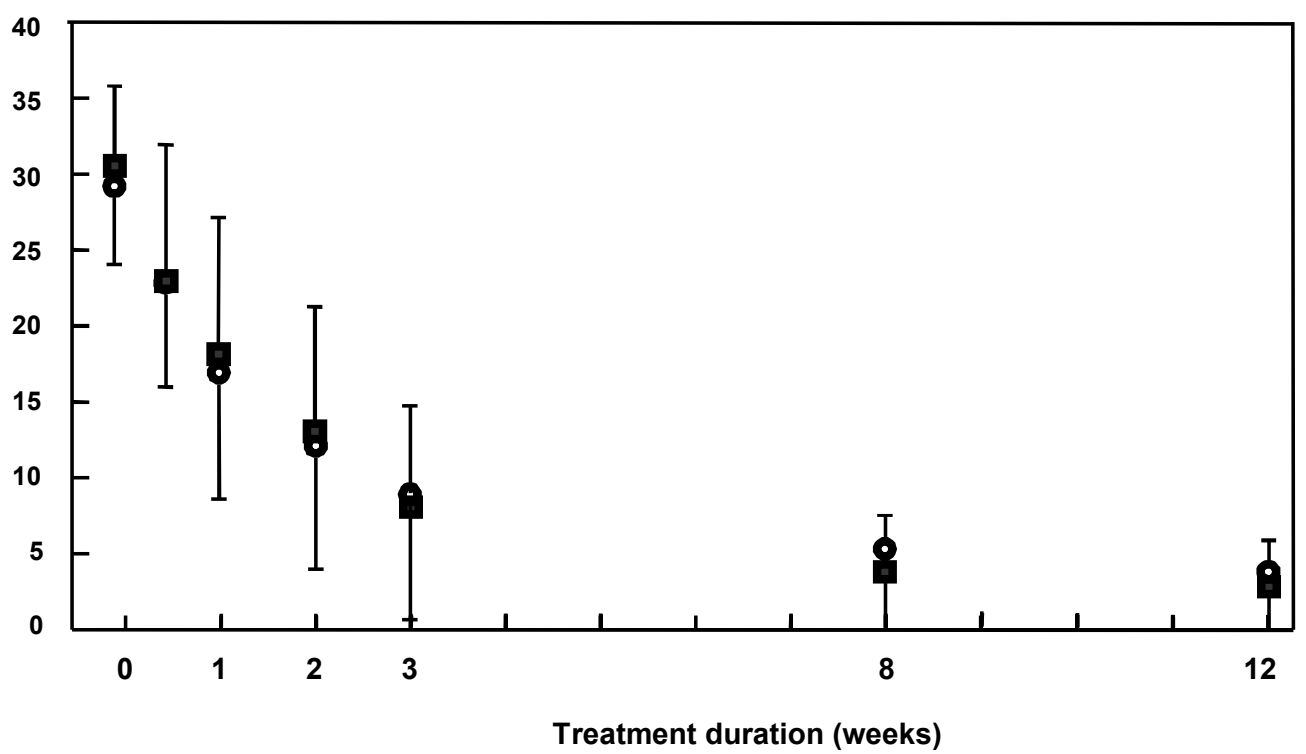

Figure 3 Young Mania Rating Scale scores over the course of the study in the intention to treat population. Data are presented as mean scores \pm standard deviation. $\bigcirc:$ amisulpride group; $\mathbf{\square}$ : haloperidol group. 
Table 3 Changes in rating scale scores over the study period in the ITT population. Data are presented as mean values \pm standard deviation of change from baseline

\begin{tabular}{|c|c|c|c|}
\hline & Amisulpride + valproate $(N=62)$ & Haloperidol + valproate $(\mathbf{N}=\mathbf{5 8})$ & p (ANCOVA) \\
\hline \multicolumn{4}{|c|}{ YMRS score (mean change) } \\
\hline Week 3 & $-20.5 \pm 8.4$ & $-22.2 \pm 9.1$ & $0.67 I$ (NS) \\
\hline Week 12 & $-23.0 \pm 10.4$ & $-25.6 \pm 9.3$ & 0.309 (NS) \\
\hline \multicolumn{4}{|c|}{ BPRS score (mean change) } \\
\hline Week 3 & $-11.3 \pm 8.7$ & $-13.7 \pm 9.3$ & $0.312(N S)$ \\
\hline Week 12 & $-12.0 \pm 11.1$ & $-13.9 \pm 10.4$ & 0.694 (NS) \\
\hline \multicolumn{4}{|c|}{ GAS score (mean change) } \\
\hline Week 12 & $34.2 \pm 20.2$ & $3 I . I \pm 2 I .1$ & 0.420 (NS) \\
\hline \multicolumn{4}{|c|}{ MADRS score (mean change) } \\
\hline Week 12 & $-3.2 \pm 8.1$ & $-1.1 \pm 9.9$ & 0.094 (NS) \\
\hline \multicolumn{4}{|c|}{ CGI-BP score (mean change) } \\
\hline Mania & $-3.6 \pm 1.3$ & $-3.8 \pm 1.2$ & 0.318 (NS) \\
\hline Depression & $0.3 \pm 1.2$ & $0.5 \pm 1.3$ & 0.269 (NS) \\
\hline General bipolar & $-2.6 \pm 1.8$ & $-3.0 \pm 1.8$ & 0.230 (NS) \\
\hline
\end{tabular}

Abbreviations: BPRS, Brief Psychiatric Rating Scale; CGI-BP, Clinical Global Impression Bipolar Sale; GAS, Global Assessment Scale; MADRS, Montgomery and Åsberg Depression Rating Scale; YMRS, Young Mania Rating Scale; NS, nonsignificant.

All patients could be discharged after 2 weeks, as specified in the protocol, with the exception of 3 patients in the haloperidol group for whom continued hospitalization was considered necessary to manage depressive symptoms. One patient in the haloperidol group required rehospitalization due to an emergent depressive mood episode while continuing medication, and 2 patients in the amisulpride group who had decided to stop treatment before the end of the study required rehospitalization due to an emergent mood episode ( 1 case of mania and 1 of depression). Two patients were rehospitalized for reasons other than mood disorders, a woman in the amisulpride group for breast cancer surgery, and a women in the haloperidol group for management of major anxiety related to extrapyramidal symptoms.

\section{Safety}

Treatment-emergent adverse events were reported in $76 \%$ of subjects overall (Table 4). Most (74.9\%) of the 203 events reported were considered to be treatment-related. These events were reported more frequently in subjects in the haloperidol group than in the amisulpride group ( $\mathrm{p}=0.009 ; \chi^{2}$ test). The most frequently reported adverse events were extrapyramidal disorders, akathisia, depression and tremor. Extrapyramidal disorders, akathisia, tremor, hypersialorrhoea, and dyskinesia were reported more frequently in subjects receiving haloperidol, whereas fatigue, somnolence, and amenorrhoea were more frequent in the amisulpride group. Severe adverse events were more frequent in the haloperidol group $(\mathrm{p}=0.004)$. Treatment was discontinued due to an adverse event in 7 subjects treated with amisulpride ( 1 case of sleepiness and 3 cases each of depression and extrapyramidal symptoms) and 15 subjects treated with haloperidol ( 9 cases of extrapyramidal symptoms, 5 cases of depression, and 1 case of rash). In 1 patient treated with haloperidol, the dose was reduced to $2.5 \mathrm{mg}$ in order to control excessive drooling and the drug subsequently discontinued due to the emergence of depression. Serious adverse events were reported in 3 subjects in the amisulpride group (1 case each of breast cancer, depression and mania) and in 5 subjects ( 1 case each of cytolytic hepatitis and anxiety and 3 cases of depression) in the haloperidol group. No severe or serious adverse events were considered to be related to treatment in the amisulpride group. No deaths were reported during the study.

Scores on the Simpson-Angus Scale increased significantly over the course of the treatment period in both groups (Table 5). However, the extent of the increase was significantly higher

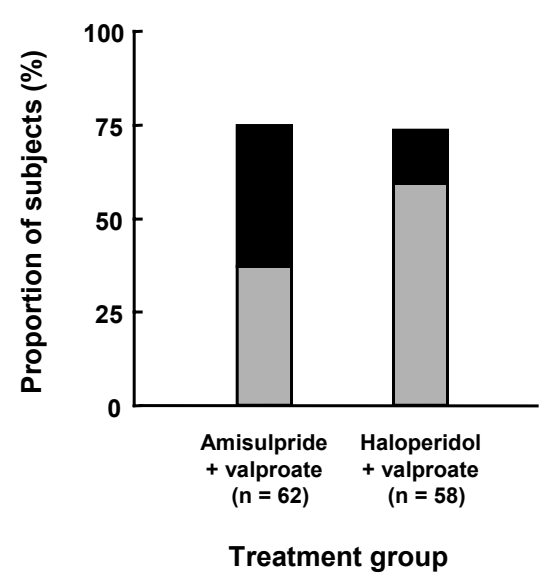

Figure 4 Improvement over the course of the study in the intention to treat population. Patient satisfaction questionnaire. Grey bars: much improved; black bars: very much improved. 
Table 4 Treatment-emergent adverse events reported in the Safety population ${ }^{a}$

\begin{tabular}{llll}
\hline & Amisulpride + valproate $(\mathbf{N}=62)$ & Haloperidol + valproate (N = 59) & $\mathbf{p}$ \\
\hline TEAEs & $41(66.1 \%)$ & $51(86.4 \%)$ & 0.009 \\
TEAEs related to study treatment & $34(54.8 \%)$ & $48(81.4 \%)$ & 0.002 \\
Severe TEAEs & $2(3.2 \%)$ & $12(20.3 \%)$ & 0.004 \\
Severe TEAEs related to study treatment & None & $12(20.3 \%)$ & $<0.001$ \\
TEAEs resulting in discontinuation & $7(11.3 \%)$ & $15(25.4 \%)$ & 0.044 \\
Serious TEAEs & $3(4.8 \%)$ & $5(8.5 \%)$ & $0.484(\mathrm{NS})$ \\
Serious TEAEs related to study treatment & None & $4(6.8 \%)$ & $0.054(\mathrm{NS})$ \\
\hline
\end{tabular}

${ }^{\mathrm{a}}$ Data are presented as number of subjects (\%).

Abbreviations: TEAEs, treatment-emergent adverse events; NS, nonsignificant.

in the haloperidol treatment group. The difference in change from baseline between the two treatment groups was -2.514 (95\% CI: $-3.965 ;-1.062]$. Similarly, the increase in score on the Barnes akathisia index was also significantly higher in the haloperidol group (Table 5). For the AIMS, an increase in mean scores was observed in the haloperidol group but not in the amisulpride group (Table 5). The difference in change from baseline between the two treatment groups for the AIMS score was -0.901 (95\% CI: $-1.737 ;-0.065)$. Antiparkinsonian drugs were considered to be required for 12 subjects in the amisulpride group and for 32 in the haloperidol group (Table 5).

Potentially clinically significant abnormalities in liver enzymes and electrolytes were observed in a minority of subjects (Table 6). Potentially significant changes in blood pressure or heart rate were observed in 15 subjects ( 7 treated with amisulpride and 8 treated with haloperidol), most commonly orthostatic hypotension (10 subjects). Clinically significant weight gain ( $>7 \%$ from baseline) was observed in 5 subjects receiving amisulpride and in two receiving haloperidol. In addition, one haloperidol-treated subject lost a significant amount of weight $(7.8 \mathrm{~kg})$. None of these findings was identified as serious adverse events and none led to treatment discontinuation.

\section{Discussion}

This randomized, controlled study in acute mania demonstrated that combination treatment with valproate and either amisulpride or haloperidol led to a treatment response rate of $72.6 \%$ and $65.5 \%$ respectively over a 12 -week study period. The incidence of adverse events in general and of extrapyramidal symptoms in particular was lower in the amisulpride group than in the haloperidol group.

The study used a composite primary efficacy outcome measure combining antimanic treatment response and study completion, because the extent of adherence to protocols in clinical trials in acute mania can be very low, with more than $50 \%$ of premature study discontinuations in some studies (Licht 2001, 2002). This renders the findings of such trials difficult to interpret, particularly when using classical methods for managing missing data such as LOCF. Restricting the analysis to subjects who complete the study thus increases the robustness of the results. Nonetheless, in the present study, discontinuation rates were relatively low, $24 \%$ in the

Table 5 Measures of extrapyramidal function in the Safety population ${ }^{\mathrm{a}}$

\begin{tabular}{|c|c|c|c|}
\hline & Amisulpride + valproate $(\mathrm{N}=62)$ & Haloperidol + valproate $(\mathbf{N}=\mathbf{5 9})$ & $\mathbf{p}$ \\
\hline \multicolumn{4}{|l|}{ Simpson-Angus Scale } \\
\hline Baseline & $0.4(0.17 ; 0.70)$ & I.I $(0.33 ; 1.94)$ & \multirow[t]{3}{*}{0.001 (ANOVA) } \\
\hline Endpoint & $1.6(0.86 ; 2.35)$ & $4.8(3.37 ; 6.26)$ & \\
\hline Change & $1.2(0.46 ; 1.87)$ & $3.7(2.38 ; 4.98)$ & \\
\hline \multicolumn{4}{|l|}{ Barnes Akathisia Index } \\
\hline Baseline & $0.0(-0.01 ; 0.10)$ & $0.2(0.05 ; 0.32)$ & \multirow[t]{2}{*}{$<0.00 \mathrm{I}$ (t-test) } \\
\hline Endpoint & $0.1(0.01 ; 0.25)$ & $0.7(0.44 ; 1.02)$ & \\
\hline \multicolumn{4}{|l|}{ AIMS score } \\
\hline Baseline & $0.3(0.04 ; 0.63)$ & $1.0(0.29 ; 1.68)$ & \multirow[t]{3}{*}{0.035 (ANOVA) } \\
\hline Endpoint & $0.2(0.0 I ; 0.4 I)$ & $1.8(0.61 ; 2.93)$ & \\
\hline Change & $-0.1(-0.35 ; 0.09)$ & $0.8(-0.08 ; 1.62)$ & \\
\hline Use of antiparkinsonian medication & $12(19.4 \%)$ & $32(54.2 \%)$ & $0.00 I$ ( $\chi^{2}$ test $)$ \\
\hline
\end{tabular}

${ }^{2}$ Data are presented as mean values with $95 \%$ confidence intervals. Abbreviation: AIMS, Abnormal Involuntary Movement Scale. 
Table 6 Potentially clinically significant abnormalities in biochemical parameters in the Safety population

\begin{tabular}{lll}
\hline & $\begin{array}{l}\text { Amisulpride }+ \\
\text { valproate } \\
(\mathbf{N}=\mathbf{6 2})\end{array}$ & $\begin{array}{l}\text { Haloperidol + } \\
\text { valproate } \\
\mathbf{( N = 5 9 )}\end{array}$ \\
\hline $\begin{array}{l}\text { Aspartate aminotransferase } \\
>2 \times \text { ULN }\end{array}$ & - & $\mathrm{I}$ \\
$\begin{array}{l}\text { Alanine aminotransferase } \\
>2 \times \text { ULN }\end{array}$ & $\mathrm{I}$ & 2 \\
$\gamma$-Glutamyltransferase & 2 & 3 \\
$>2 \times$ ULN & - & $\mathrm{I}$ \\
$\begin{array}{l}\text { Alkaline phosphatase } \\
>\text { I.5 } \times \text { ULN }\end{array}$ & & $\mathrm{I}$ \\
$\begin{array}{l}\text { Potassium }<3 \text { mmol/L or } \\
\geq 5.5 \mathrm{mmol} / \mathrm{L}\end{array}$ & - & 3 \\
$\begin{array}{l}\text { Total bilirubin }>\text { ULN } \\
\text { Conjugated bilirubin }>\text { ULN }\end{array}$ & 8 & 4 \\
\hline
\end{tabular}

Abbreviation: ULN, upper limit of normal.

amisulpride group and 38\% in the haloperidol group. Since the emergence of adverse events was the principal driver of nonadherence, the better-tolerated amisulpride was associated with better adherence. This has important consequences for effectiveness of treatment in everyday clinical practice where patient follow-up may be less intensive than in a randomized clinical trial.

The response rate in the haloperidol group from this study was similar to that observed in the combination treatment group $(70 \%)$ in a previous study of haloperidol or perazine combined with valproate versus valproate alone (Muller-Oerlinghausen et al 2000). The decrease in YMRS score observed was rather larger than that reported for the haloperidol group in a study of adjunctive risperidone versus haloperidol in combination with lithium or valproate (Sachs et al 2002). Treatment responses in the amisulpride and valproate group were of comparable magnitude to those reported in previous trials of other atypical antipsychotics combined with lithium or valproate in the treatment of acute mania (Sachs et al 2002, 2004; Tohen et al 2002; Yatham et al 2004).

Comparable efficacy between the two treatment groups was observed across all efficacy end-points evaluated, supporting the robustness and consistency of the findings. Although superiority of amisulpride over haloperidol was not demonstrated, both drugs had notably high response rates. In a comparative study of olanzapine and haloperidol (Tohen et al 2003), olanzapine did not differ from haloperidol in achieving overall remission of bipolar mania. In another monotherapy trial, comparing aripiprazole with haloperidol, the atypical antipsychotic did prove to be superior using the same measure of effectiveness as this one (Vieta et al 2005a). However, in this study anticholinergic medication was not allowed and this caused a high number of drop-outs in the haloperidol arm, thus reducing the number of patients achieving the end-point.

No difference between treatments in terms of switch to depression or relapse into mania was observed, which concerned a relatively small number of subjects (no more than 10 per group). However, the frequency of switch to depression appeared to be in favor of the amisulpride group (11.3\% vs $19.0 \%)$. Switch to depression in either treatment group was independent of baseline MADRS depression symptoms scores. Given the potential antidepressant effect of amisulpride observed in other patient groups (Montgomery 2002; Peuskens et al 2002), this merits a dedicated study in bipolar depression.

The difference between the two treatment groups in terms of extrapyramidal symptoms is consistent with what is known of the comparative tolerability of amisulpride and haloperidol in schizophrenia. Antiparkinsonian medication was required by $55.2 \%$ of subjects receiving haloperidol compared to $19.4 \%$ of those receiving amisulpride. These data can be compared with the proportions of patients requiring such medication in the risperidone (17\%) and haloperidol (38\%) arms of the earlier valproate combination study of these drugs (Sachs et al 2002). There was no obvious specific tolerability issue associated with amisulpride treatment. The adverse event profile of amisulpride was consistent with that described in clinical trials in patients with schizophrenia (Rein et al 2000). Overall, the observation of similar efficacy to haloperidol, with improved tolerability, is consistent with data from clinical trials comparing other atypical antipsychotics to haloperidol in acute mania (Scherk et al 2007).

Concerns have been raised about the poor generalizability of many trials in the field of bipolar disorder (Vieta and Carne 2005). This trial tried to overcome such concerns with a pragmatic design which imposed fewer constraints on patients and stipulated less stringent entry criteria. Anticholinergic drugs were thus allowed, and all patients received valproate. Nonetheless, inspection of the characteristics of included patients revealed that the patients were typically middle-aged, with a mean duration of illness of 12.6 years and experiencing relatively frequent and recent mood episodes. Typically, patients had been previously treated with, and presumably responded to, valproate rather than lithium. It will be important to determine whether the results can be replicated in patients who are responsive to lithium, in 
patients with earlier disease, and in patients not treated with a mood stabilizer.

The study has a number of limitations. Firstly, the absence of a placebo group precludes estimation of the absolute size of the treatment effect. However, the adoption of a placebo arm may limit inclusion into the study to less severely ill patients and thus restrict the generalizability of the findings (Vieta and Carne 2005). This limitation needs to be addressed in future studies by comparing outcome in patients receiving mood stabilizers alone with that in patients treated with mood stabilizers and adjunctive amisulpride treatment. Secondly, the open design introduces possible bias from the expectations of patients or investigators, although randomization should to some extent reduce the potential sources of bias inherent in open studies. Thirdly, although it matched what was anticipated in the a priori power calculations, the sample size was rather small, particularly considering the rates of premature study discontinuation $(31.7 \%)$ and of major protocol violations (15.0\%). For this reason, potentially relevant trends towards inter-group differences in secondary outcome measures, such as relapse into a new manic episode or change in depressive symptoms, could not be confirmed. In future studies of this type, it would be important to include more patients per group in order to address a wider variety of outcome measures. A related limitation concerns the duration of the study. Although a 12-week duration conforms to contemporary methodological guidelines for clinical trial design in acute mania (European Medicines Agency 2007) and seems justified in an initial study, it does not allow the potential long-term benefit of treatment in preventing future mood episodes. A longer study duration would be necessary to confirm or refute the observed trend towards more effective relapse prevention in patients receiving amisulpride.

To our knowledge, this is the first randomized comparative study of amisulpride for the treatment of acute mania. Although the size and goals of the study were relatively modest, it yielded encouraging information on the potential benefit provided by amisulpride in this patient population and opens perspectives for future studies aimed at clarifying the role of this antipsychotic in the treatment of mania. As well as benchmarking the treatment effect size for monotherapy with mood stabilizers, or possibly for other atypical antipsychotic drugs, such studies should address the use of amisulpride for preventing manic relapse, efficacy for functional outcome, and quality of life, collection of data for relatives or carers, and possible differential efficacy in different patient groups.
In conclusion, this study suggests that combination treatment with amisulpride and valproate represents a relatively effective and well-tolerated treatment option for acute mania.

\section{Acknowledgments}

The authors wish to thank the principal investigators of all participating study centers and their teams for their enthusiastic contribution to this project:

Czech Republic: Dr J Tomanova (Brno-Bohunice); Dr L Hosak (Hradec Králové); Dr D Seifertova (Praha 8-1); Dr T Petranova (Plzen-Lochotín); Dr J Boucek (Olomouc); Dr V Hanuskova (Opava); Dr P Navratil (Praha 6); Dr V Burian (Tabor); Dr L Macak (Tabor). France: Pr P Thomas (Lille); Dr G Ruetsch (Poitiers); Dr J-A Meynard (La Rochelle); Dr M Cazenave (Bordeaux); Dr J Louvrier (Bully Les Mines); Dr GP Badet (Dijon); Dr M Abbar (Nîmes); Dr J-C Samuelian (Marseille); Dr J Palazzolo (Nice); Dr B Millet (Rennes); Dr G Allio (Sotteville Les Rouen). Poland: Dr I Krupka Matuszczyk (Katowice); Dr H Marmurowska (Lublin); Dr J Landowski (Gdansk); Dr M Janiszewski (Torun); Dr A Przewlocka (Krakow); Dr J Bukowski (Gorlice). Slovakia: Dr P Cernak (Pezinok); Dr E Palova (Kosice); Dr L Nabelek (Banská Bystrica); Dr L. Vavrusova (Bratislava); Dr V Novotny (Bratislava); Dr H Shahpesandi (Liptovsky Mikulas); Dr Z Janikova (Liptovsky Mikulas); Dr M Halmo (Bratislava - Petrzalka); Dr V Garaj (Bojnice); Dr L Vircik (Michalovce). Spain: Dr MA Gonzales Torres (Bilbao); Dr E Vieta (Barcelona); Dr E Alvarez (Barcelona); Dr A Carrasco (Ciudad Real).

\section{Disclosures}

This study was sponsored and funded by Sanofi-Aventis, manufacturers of amisulpride and of sodium valproate. Operational management of the study (study monitoring, data collection, and data analysis) was delegated by ICTA PM, a contract research association.

The authors made up the Steering Committee for the trial. They advised the study sponsor on the design of the protocol and the implementation of the study, reviewed the results, made suggestions for further analyses, and participated in the interpretation of the data. Both authors received honoraria from the sponsor for their involvement in the study as well as consultancy fees for advice in the field of psychiatry in general over the previous 3 years. The corresponding author, PT, had full access to all data from the study, and had final responsibility for the decision to submit the finalized manuscript for publication. 


\section{References}

Angst J, Angst F, Gerber-Werder R, et al. 2005. Suicide in 406 mooddisorder patients with and without long-term medication: a 40 to 44 years' follow-up. Arch Suicide Res, 9:279-300.

[APA] American Psychiatric Association. 2002. Practice guideline for the treatment of patients with bipolar disorder (revision). Am J Psychiatry, 159:1-50

[APA] American Psychiatric Association. 2000. Diagnostic and Statistical Manual of Mental Disorders. Washington DC: APA.

Barnes TR. 1989. A rating scale for drug-induced akathisia. Br J Psychiatry, 154:672-6.

Bowden CL. 2005. Atypical antipsychotic augmentation of mood stabilizer therapy in bipolar disorder. J Clin Psychiatry, 66(Suppl 3):12-9.

Carta M, Zairo F, Mellino G, et al. 2006. An open label follow-up study on amisulpride in the add-on treatment of bipolar I patients. Clin Pract Epidemol Ment Health, 2:19.

Elgie R, Morselli PL. 2007. Social functioning in bipolar patients: the perception and perspective of patients, relatives and advocacy organizations - a review. Bipolar Disord, 9:144-57.

Endicott J, Spitzer RL, Fleiss JL, et al. 1976. The global assessment scale. A procedure for measuring overall severity of psychiatric disturbance. Arch Gen Psychiatry, 33:766-71.

European Medicines Agency. 2007. Note for guidance on clinical investigation of medical products in the treatment and prevention of bipolar disorder.

Goodwin GM. 2003. Evidence-based guidelines for treating bipolar disorder: recommendations from the British Association for Psychopharmacology. J Psychopharmacol, 17:149-73; discussion 147.

Grunze H, Kasper S, Goodwin G, et al. 2003. The World Federation of Societies of Biological Psychiatry (WFSBP) Guidelines for the Biological Treatment of Bipolar Disorders, Part II: Treatment of Mania. World J Biol Psychiatry, 4:5-13.

[NIMH] National Institute of Mental Health. 1976. AIMS, Abnormal Involuntary Movement Scale. In Guy W (ed.). ECDEU Assessment Manual of Psychopharmacology Review. Rockville, USA: NIMH.

Krishnan KR. 2005. Psychiatric and medical comorbidities of bipolar disorder. Psychosom Med, 67:1-8.

Leucht S, Pitschel-Walz G, Engel RR, et al. 2002. Amisulpride, an unusual "atypical" antipsychotic: a meta-analysis of randomized controlled trials. Am J Psychiatry, 159:180-90.

Levin FR, Hennessy G. 2004. Bipolar disorder and substance abuse. Biol Psychiatry, 56:738-48.

Licht RW. 2001. Limitations in randomised controlled trials evaluating drug effects in mania. Eur Arch Psychiatry Clin Neurosci, 251(Suppl 2): II66-71.

Licht RW. 2002. Limits of the applicability and generalizability of drug trials in mania. Bipolar Disord, 4(Suppl 1):66-8.

McIntyre RS, Brecher M, Paulsson B, et al. 2005. Quetiapine or haloperidol as monotherapy for bipolar mania - a 12-week, doubleblind, randomised, parallel-group, placebo-controlled trial. Eur Neuropsychopharmacol, 15:573-85.

Montgomery SA. 2002. Dopaminergic deficit and the role of amisulpride in the treatment of mood disorders. Int Clin Psychopharmacol, 17(Suppl 4):S9-15; discussion S16-7.

Montgomery SA, Åsberg M. 1979. A new depression scale designed to be sensitive to change. Br J Psychiatry, 134:382-9.

Muller-Oerlinghausen B, Retzow A, Henn FA, et al. 2000. Valproate as an adjunct to neuroleptic medication for the treatment of acute episodes of mania: a prospective, randomized, double-blind, placebo-controlled, multicenter study. European Valproate Mania Study Group. J Clin Psychopharmacol, 20:195-203.
Overall J, Gorham D. 1962. The Brief Psychiatric Rating Scale. Psychol Reports, 10:799-812.

Peuskens J, Moller HJ, Puech A. 2002. Amisulpride improves depressive symptoms in acute exacerbations of schizophrenia: comparison with haloperidol and risperidone. Eur Neuropsychopharmacol, 12:305-10.

Pini S, de Queiroz V, Pagnin D, et al. 2005. Prevalence and burden of bipolar disorders in European countries. Eur Neuropsychopharmacol, 15:425-34.

Rein W, Coulouvrat C, Dondey-Nouvel L. 2000. Safety profile of amisulpride in short- and long-term use. Acta Psychiatr Scand Suppl, 400:23-7.

Sachs G, Chengappa KN, Suppes T, et al. 2004. Quetiapine with lithium or divalproex for the treatment of bipolar mania: a randomized, doubleblind, placebo-controlled study. Bipolar Disord, 6: 213-23.

Sachs GS, Grossman F, Ghaemi SN, et al. 2002. Combination of a mood stabilizer with risperidone or haloperidol for treatment of acute mania: a double-blind, placebo-controlled comparison of efficacy and safety. Am J Psychiatry, 159:1146-54.

Scherk H, Pajonk FG, Leucht S. 2007. Second-generation antipsychotic agents in the treatment of acute mania: a systematic review and meta-analysis of randomized controlled trials. Arch Gen Psychiatry, 64:442-55.

Segal J, Berk M, Brook S. 1998. Risperidone compared with both lithium and haloperidol in mania: a double-blind randomized controlled trial. Clin Neuropharmacol, 21:176-80.

Shi L, Namjoshi MA, Zhang F, et al. 2002. Olanzapine versus haloperidol in the treatment of acute mania: clinical outcomes, health-related quality of life and work status. Int Clin Psychopharmacol, 17:227-37.

Simpson G, Angus J. 1970. A rating scale for extrapyramidal symptoms. Acta Psychiatr Scand, 45:11-19.

Smith LA, Cornelius V, Warnock A, et al. 2007. Pharmacological interventions for acute bipolar mania: a systematic review of randomized placebo-controlled trials. Bipolar Disord, 9:551-60.

Smulevich AB, Khanna S, Eerdekens M, et al. 2005. Acute and continuation risperidone monotherapy in bipolar mania: a 3-week placebo-controlled trial followed by a 9-week double-blind trial of risperidone and haloperidol. Eur Neuropsychopharmacol, 15:75-84.

Spearing MK, Post RM, Leverich GS, et al. 1997. Modification of the Clinical Global Impressions (CGI) Scale for use in bipolar illness (BP): the CGI-BP. Psychiatry Res, 73:159-71.

Tohen M, Chengappa KN, Suppes T, et al. 2002. Efficacy of olanzapine in combination with valproate or lithium in the treatment of mania in patients partially nonresponsive to valproate or lithium monotherapy. Arch Gen Psychiatry, 59:62-9.

Tohen M, Goldberg JF, Gonzalez-Pinto Arrillaga AM, et al. 2003. A 12 -week, double-blind comparison of olanzapine vs haloperidol in the treatment of acute mania. Arch Gen Psychiatry, 60:1218-26.

Vieta E, Bourin M, Sanchez R, et al. 2005a. Effectiveness of aripiprazole v. haloperidol in acute bipolar mania: double-blind, randomised, comparative 12-week trial. Br J Psychiatry, 187:235-42.

Vieta E, Carne X. 2005. The use of placebo in clinical trials on bipolar disorder: a new approach for an old debate. Psychother Psychosomatics, $74: 10-16$.

Vieta E, Ros S, Goikolea JM, et al. 2005b. An open-label study of amisulpride in the treatment of mania. J Clin Psychiatry, 66:575-8.

Yatham LN, Paulsson B, Mullen J, et al. 2004. Quetiapine versus placebo in combination with lithium or divalproex for the treatment of bipolar mania. J Clin Psychopharmacol, 24:599-606.

Young RC, Biggs JT, Ziegler VE, et al. 1978. A rating scale for mania: reliability, validity and sensitivity. Br J Psychiatry, 133:429-35. 
